\title{
Delivery of antagomiR204-conjugated gold nanoparticles from PLGA sheets and its implication in promoting osseointegration of titanium implant in type 2 diabetes mellitus
}

\author{
This article was published in the following Dove Press journal: \\ International Journal of Nanomedicine \\ 26 September 2017 \\ Number of times this article has been viewed
}

\author{
Xiangwei Liu, ${ }^{\text {, } * ~ N a i w e n ~}$ \\ Tan, ${ }^{1, *}$ Yuchao Zhou, ' \\ Hongbo Wei, ${ }^{1, *}$ Shuai \\ Ren, ' Fan Yu, ${ }^{2}$ Hui Chen, ${ }^{3}$ \\ Chengming Jia, ${ }^{4}$ Guodong \\ Yang, ${ }^{5}$ Yingliang Song' \\ 'State Key Laboratory of Military \\ Stomatology \& National Clinical \\ Research Center for Oral Diseases \& \\ Shaanxi Engineering Research \\ Center for Dental Materials and \\ Advanced Manufacture, Department \\ of Implant Dentistry, ${ }^{2}$ Department \\ of Prosthodontics, School of \\ Stomatology, ${ }^{3}$ Department of Plastic \\ Surgery, Tangdu Hospital, ${ }^{4}$ Department \\ of Traditional Chinese Medicine, \\ Xijing Hospital, ${ }^{5}$ Department of \\ Biochemistry and Molecular Biology, \\ Fourth Military Medical University, \\ Xi'an, China \\ *These authors contributed equally \\ to this work
}

\begin{abstract}
Impaired osseointegration of the implant remains the big hurdle for dental implant therapy in diabetic patients. In this study, the authors first identified that miR204 was strikingly highly expressed in the bone mesenchymal stem cells (BMSCs) of diabetic rats. Forced expression of miR204 repressed the osteogenic potential of BMSCs, while inhibition of miR204 significantly increased the osteogenic capacity. Moreover, the miR204 inhibitor was conjugated with gold nanoparticles (AuNP-antagomiR204) and dispersed them in the poly(lactic-co-glycolic acid) (PLGA) solution. The AuNP-antagomiR204 containing PLGA solution was applied for coating the surface of titanium implant. Electron microscope revealed that an ultrathin sheet was formed on the surface of the implant, and the AuNPs were evenly dispersed in the coated PLGA sheet. Cellular experiments revealed that these encapsulated AuNP-antagomiR204 were able to be released from the PLGA sheet and uptaken by adherent BMSCs. In vivo animal study further confirmed that the AuNP-antagomiR204 released from PLGA sheet promoted osseointegration, as revealed by microcomputerized tomography (microCT) reconstruction and histological assay. Taken together, this study established that miR204 misexpression accounted for the deficient osseointegation in diabetes mellitus, while PLGA sheets aided the release of AuNP-antagomiR204, which would be a promising strategy for titanium implant surface functionalization toward better osseointegration.
\end{abstract}

Keywords: osseointegration, miR204, PLGA sheets, gold nanoparticles, diabetes mellitus, titanium implant

\section{Introduction}

Dental implant is choice of the first priority for numerous patients with missing teeth, due to its comfort, efficiency, and requiring no adjacent teeth adjustment. The most commonly used titanium implant achieves at least 95\% success rate in the general population, ${ }^{1,2}$ while the success rate is compromised in patients with diabetes mellitus (DM) ${ }^{3,4}$ According to a recent survey, there are $\sim 415$ million people suffering from DM in 2015, ${ }^{5}$ among which type 2 diabetes mellitus (T2DM) accounts for $>90 \%{ }^{6}$ Although numerous studies have shown that the poor outcome associated with DM might be attributed to high glucose environment, microangiopathy, and bone matrix secretion inability of osteoblasts, ${ }^{7,8}$ the detailed mechanism of how DM interferes with osseointegration of dental implant is not fully understood.

Although therapy of DM itself would yield better osseointegration, the long-lasting harmful effects of DM are to some extent irreversible and difficult for correction..$^{9,10}$
Correspondence: Guodong Yang Molecular Biology, Fourth Military ' Email yanggd@fmmu.edu.cn

Yingliang Song

Department of Implant Dentistry, School of Stomatology, Fourth Military Medical University, No 145 West Changle Road, Xi'an, China

Tel +86298477 6454

Email yingliangsong@163.com 
Surface processing of dental implant, such as microarc oxidation (MAO) and acid-etching, provides another option for bettering osseointegration, which has significantly improved the bone-implant contact (BIC) and gained extensive attention in clinical implant areas. ${ }^{1,11}$ However, simply having the surface handled with physical changes is not enough for patients with DM. Bone mesenchymal stem cells (BMSCs) are the key cell components responsible for osteogenesis. It has been reported that BMSC suspension or cell sheets were manipulated to modify the implant for better outcome. ${ }^{12}$ However, success was achieved with great difficulty due to the complex procedure, few survived cells, and poor primary stability. In situ and in vivo delivery of factors regulating biological processes of ossification have been proved to promote wound healing and tissue regeneration. ${ }^{13,14}$ MicroRNAs (miRNAs) are 19-25 nucleotide long non-coding RNAs. By binding to a partially complementary sequence of the target messenger RNAs (mRNAs) and subsequently silencing them, miRNAs are found to participate in multiple cellular functions, such as cell proliferation, differentiation, adhesion, and migration. ${ }^{15,16}$ It is widely accepted that dysregulation of osteogenesis-related genes is responsible for the poor healing performance of bone injury in T2DM, osteoporosis, or other systemic diseases patients. ${ }^{17-19}$ In this regard, certain osteogenesis-related miRNAs would be expected to be involved, while very little work has been done in this field, especially in the context of bone regeneration and oral implantation. It has been reported that miR204 acts as a negative regulator during BMSC osteogenic differentiation by directly repressing runt-related transcription factor 2 (Runx2), ${ }^{20}$ a key transcription factor in osteogenesis.

In this study, the expression and putative role of miR204 in osteogenesis in the context of hyperglycemia were first explored. Compared with the expression in BMSCs from normal rats, miR204 was much higher in cells from T2DM rats. Inhibition of miR204 significantly improved the osteogenesis of BMSCs from diabetic rats. Moreover, antagomiR204 (miR204 inhibitor)-conjugated gold nanoparticles could be evenly dispersed in the PLGA solution, and the mixture was used to form a stable ultrathin film on the titanium implant. The resultant modified implant produced a significantly better outcome of osseointegration. This study provides a novel titanium implant surface processing strategy for better osseointegration.

\section{Materials and methods}

\section{Preparation of T2DM rat model}

Sprague Dawley (SD) rats (male, 12 months old) obtained from the animal center of the Fourth Military Medical
University (FMMU) were grown and processed in accordance with the Animal Handling Guideline of FMMU and all animal procedures were approved by the Institutional Animal Care and Use Committee (IACUC) of FMMU. For the preparation of T2DM model, SD rats that received 1-week adaptability and underwent 12-hour fasting were intraperitoneally injected with $1 \%$ streptozotocin (30 mg/100 g; Sigma, St Louis, MO, USA) dissolved in citrate buffer $(\mathrm{pH}, 4.2)$ for two times every other day. Later, high-fat refined-sugar diet was provided until the research was completed. The glucose level was monitored regularly every week, and the rats with relatively stable blood glucose level between 16.7 and $25.6 \mathrm{mmol} / \mathrm{L}$ were considered as T2DM rats and selected for the next experiments.

\section{Cell isolation and culture}

For the isolation of BMSCs, the rats were sacrificed by euthanization followed by cervical dissection. The bone marrows of bilateral femur and tibia were harvested with the aid of syringe under sterile conditions. Cells were pelleted and resuspended for culture in DMEM medium (Gibco, Waltham, MA, USA) containing 10\% fetal bovine serum (Gibco) and 1\% antibiotics (Hyclone, Logan, UT, USA) in humidified atmosphere of $5 \% \mathrm{CO}_{2}$ at $37^{\circ} \mathrm{C}$. The BMSCs were passaged when $90 \%$ confluence was reached, and passage 2 cultures were used in this study.

\section{Mineralization induction and staining}

For mineralization induction, $70 \%$ confluent cells were induced by osteogenic induction medium (Cyagen, Guangzhou, China) changed every 3 days. After 7-day osteogenic induction, the alkaline phosphatase (ALP) activity assay was performed by using ALP assay kit according to the instructions (Beyotime, Jiangsu, China). The full osteogenesis was achieved by 3 -week induction and examined by alizarin red (Sigma) staining of mineralized matrix. The induced mineralization nodules were quantified by dissolving the adherent alizarin red in cetylpyridinium chloride (Sigma) and subsequent optical density value measurement was $570 \mathrm{~nm}$.

\section{Real-time quantitative polymerase chain reaction (PCR) analysis}

Total RNAs from cells or tissues were isolated by using the Trizol (Thermo Fisher Scientific, Waltham, MA, USA), while miRNA was further isolated with miRNA isolation kit (Ambion, Carlsbad, CA, USA). Isolated RNAs were then subjected to reverse transcription using PrimeScript 
RT Reagent kit (Takara, Shiga, Japan) or microRNA RT kit (Tiangen, Brijing, China). Real-time quantitative PCR (qPCR) analysis was conducted with SYBR Premix Ex Taq II (Takara) by using a CFX96 Real Time System (Bio-Rad, Hercules, CA, USA). Expression of miR204 and osteogenesis-related genes including Bmp, Opg, Alp, Runx2, and Col 1 were detected. Gapdh and RNU6 were selected as the internal control for mRNA and miRNA expression, respectively. The referred primer sequences are listed in Table 1.

\section{Conjugation of antagomiRNAs with gold nanoparticles}

AntagomiRNAs were conjugated to gold nanoparticles as described before. ${ }^{21}$ Briefly, $50 \mu \mathrm{L}$ antagomiRNAs $(30 \mu \mathrm{mol} / \mathrm{L}$, 5'-labeled with Cy3 or not, 3'-modified with thiol; Ribbio, Guangzhou, China) was dissolved in $450 \mu \mathrm{L}$ of phosphatebuffered saline (PBS, $\mathrm{pH}=8.5$ adjusted with $\mathrm{NaOH}$ ) and then processed by dithiothreitol (Takara) to reduce the disulfide bond, followed by purification. Then, $24 \mu \mathrm{L}$ of nucleic acid solution was mixed with gold nanoparticles (10 nm in diameter; Sigma) in $1.5 \mathrm{~mL}$ of citrate buffer and allowed to stand for 1 hour. PBS (0.1 M, pH =7.0) was added stepwise in $10 \mu \mathrm{L}$ increment with 5-minute interval until the final concentration of sodium reached $10 \mathrm{mM}$. $\mathrm{NaCl}$ solution was also added stepwise with the same increment and interval to reach a concentration of $100 \mathrm{mM}$ before incubation at $4^{\circ} \mathrm{C}$ for 16 hours. The oligonucleotide gold nanoparticle conjugate was harvested by centrifuging at $17,000 \times g$ for 30 minutes.

\section{Construction of PLGA sheet-based AuNP-antagomiRNA delivery system}

PLGA (0.2 g, 65:35; Sigma) was dissolved completely in $2 \mathrm{~mL}$ dichloromethane (DCM; Fuyu, Tianjin, China) with a magnetic stirrer (Sanli, Hangzhou, China). Then, the PLGA solution was poured into the glass bottle containing $2 \mathrm{nmol}$ gold nanoparticle-conjugated miRNA inhibitor or control. Then, the MAO titanium implants or discs (the Northwest Institute for Nonferrous Metal Research, Xi'an, China) were immersed into the solution and taken out immediately. DCM evaporated rapidly and a thin layer of PLGA film containing miR204-inhibitor-conjugated gold nanoparticle (AuNP) was expected to be found on the surface of the samples. All the procedure was done in the chemical hood, and the DCM was removed by evaporation.

The surface morphology of the functionalized implant was analyzed by scanning electron microscopy (SEM, Hitachi S-4800; Hitachi Ltd., Tokyo, Japan). The distribution of miRNA-inhibitor-conjugated gold nanoparticles in the PLGA membrane was investigated by transmission electron microscope (TEM, JEM1230, JEOL Ltd., Tokyo, Japan) and laser scanning confocal microscope (LSCM, ECLIPSE Ti; Nikon, Tokyo, Japan). The obtained samples were sterilized by $\mathrm{Co} 60$ irradiation and stored in $4^{\circ} \mathrm{C}$ before in vivo use.

\section{Analysis of the release and uptake of the AuNP-miRNA from PLGA sheets}

For the deliverability measurement of the constructed PLGA sheets delivery system, $2 \times 10^{4}$ BMSCs derived from T2DM $\left(\mathrm{BMSCs}^{-\mathrm{T} 2 \mathrm{DM}}\right.$ ) obtained as described above were seeded on the functionalized titanium disc (covered with PLGAAuNP-Cy3 labeled antagomiR204) placed in a 24-well plate. After 24 hours of co-incubation, the cells were fixed with paraformalin and the nuclei were labeled with Hoechst (Thermo Fisher Scientific), followed by further observation with LSCM to evaluate the uptake efficiency of AuNPantagomiR204 distributed in PLGA sheets.

Table I Sequences of primers, miR204, and antagomiR204 and the controls used in the study

\begin{tabular}{lll}
\hline Name & Forward & Reverse \\
\hline Bmp & ATCGTTACCTCAAGGGAGTGG & GCGACGGCAGTTCTTATTCT \\
Opg & ACCATGTACCGATTGTATC & CTGCCATTTCAAGAGCC \\
Alp & ACAACCTGACTGACCCTTCCC & CAATCCTGCCTCCTTCCACTA \\
Runx2 & GAACAGTAGCGGGAGCAT & CCCACATAGGAGGAGAAAA \\
Coll & AAGGCAATGCTGAATCGTCC & TGGTTAGGCTCCTTCAATAGTCC \\
Gapdh & GGTGCTGAGTATGTCGTGGAG & GCGGAGATGATGACCCTTTT \\
Rnu6b & CTCGCTTCGGCAGCACA & AACGCTTCACGAATTTGCGT \\
\hline AntagomiRNAs & Sequence & \\
\hline AntagomiRScramble & 5'-rArUrC rGrArArUrUrCrCrUrGrCrArGrCrCrCrGrUrU (Spacer I8)-Thiol-3' \\
Cy3-antagomiRScramble & 5'-Cy3-rArUrC rGrArArUrUrCrCrUrGrCrArGrCrCrCrGrUrU (Spacer I8)-Thiol-3' $^{\prime}$ \\
AntagomiR204 & 5'-rGrGrCrArUrArGrGrArUrGrArCrArArArGrGrGrArA (Spacer I8)-Thiol-3' \\
Cy3-antagomiR204 & 5'-Cy3-rGrGrCrArUrArGrGrArUrGrArCrArArArGrGrGrArA (Spacer I8)-Thiol-3' \\
\hline
\end{tabular}

Abbreviations: Bmp, bone morphogenetic protein; Opg, osteoprotegerin; AIP, alkaline phosphatase; Runx2, runt-related transcription factor 2; Col I, collagen type I; Gapdh, glyceraldehyde-3-phosphate dehydrogenase; Rnu6b, RNA U6 small nuclear 2; antagomiR204, microRNA204 inhibitor. 


\section{Cell adhesion assay and cytotoxicity assay}

For cell adhesion analysis, $\sim 5 \times 10^{4} \mathrm{BMSCs}^{\mathrm{T} 2 \mathrm{DM}}$ rats were seeded onto miR204-inhibitor and control functionalized titanium discs ( $1.5 \mathrm{~cm}$ in diameter) for 4 hours, and the non-adhesive cells were washed away by PBS. BMSCs ${ }^{- \text {T2DM }}$ attached to the discs were stained with Hoechst (Thermo Fisher Scientific) and then observed and counted with LSCM.

For the cytotoxicity assay of the constructed miRNA delivery system, $2 \times 10^{4}$ BMSCs in $800 \mu \mathrm{L}$ of DMEM complete medium were seeded on titanium discs placed in 24-well plates and incubated for the indicated time. An amount of $200 \mu \mathrm{L}$ of cell counting kit 8 (CCK8) reagents (Dojindo, Kumamoto, Japan) was gently added and mixed evenly into each well at the end of culture. After 2 hours of incubation at $37^{\circ} \mathrm{C}, 200 \mu \mathrm{L}$ of the solution was transferred to a 96 -well plate, and the absorbance value was read at $490 \mathrm{~nm}$ (Bio-Rad).

\section{Placement of modified implants}

Male SD rats with T2DM were intraperitoneally injected with $1 \%$ pentobarbital $(0.4 \mathrm{~mL} / 100 \mathrm{~g}$; Xitang, Shanghai, China) and then subjected to local anesthesia with primacaine (Pierre Rolland, Bordeaux, France). The surgical field was shaved and sterilized with iodophors, and 1.5 -cm-long incisions were made at the proximal part of the bilateral tibia. Using a dental implant appliance case, perforating planting holes sized $2.8 \mathrm{~mm}$ in diameter were drilled for placed implants $(3 \times 8 \mathrm{~mm}$, MAO titanium implants), with saline irrigation cooling. The rats were randomly divided into three groups: 1) control group, rats received original implant; 2) PLGA-control, rats received implant with PLGA sheet containing scramble sequence-conjugated AuNP; and 3) PLGA $^{\text {-inhibitor }}$, rats received implant with PLGA sheet containing miR204-inhibitor-conjugated AuNP. Following suture and postoperative antiinflammation treatment with gentamycin (Humanwell, Hubei, China), animals were kept for 8 weeks recovery before osseointegration strength test, microcomputerized tomography (microCT), and histological analysis.

\section{Removal torque test}

The removal torque test was conducted immediately after the implants together with the tibias were harvested. The tests were performed with a torque meter (Aigu, Hong Kong, China), and torque applied on the implants was increased gradually after the samples fixed well. As the strength went up, the adhesion between implants and its host bodies was destroyed. The torque value at the critical moment was recorded as the removal torque value of the implant.

\section{MicroCT scanning and reconstruction}

After intraperitoneally injected with excessive pentobarbital, rats were sacrificed, and the partial tibia bone around the implant was excised and fixed in 4\% paraformaldehyde. For further imaging examination, a high-resolution microCT scanner (Yxlon, Hamburg, Germany) was employed. Bone samples were scanned, and implants with $200 \mu \mathrm{m}$ bone thickness surrounding the implants were reconstructed by using data analysis software. Bone volume fraction, number of trabecular, and trabecular thickness were calculated to assess the osseointegration.

\section{Histological analysis}

For histological analysis of osteo-implant interface after 8 weeks recovery, the undecalcified specimens were embedded in resin and then cut into $50-\mu \mathrm{m}$ sections. The sections were stained with Van-Gieson to highlight the different tissues. The digital section images were captured with the aid of light microscope (Olympus, Tokyo, Japan), and the BIC rate (the percentage of BIC zone in total implant surface size) was determined by using computer-based image analysis technique to assess the osteogenic activity of implants received different treatments.

\section{Statistical analysis}

All data are expressed as mean \pm SD. Statistical significance was determined with Student's $t$-test or analysis of variance by using SPSS software. Statistical significance was accepted for $P<0.05$.

\section{Results}

\section{miR204 inhibits matrix mineralization}

Deficient osteogenic capacity of BMSCs in DM is considered as one of the causes for impaired osseointegration. Consistent with previous research studies, ${ }^{22,23}$ this study also confirmed that $\mathrm{BMSCs}^{-\mathrm{T} 2 \mathrm{DM}}$ had inferior osteogenic capacity compared with normal BMSCs, as seen from alizarin red staining and the subsequent quantification measurement (Figure 1A and B). To explore whether the osteogenic inhibitor miR204 contributed to the poor performance of $\mathrm{BMSC}^{-\mathrm{T} 2 \mathrm{DM}}$ in osteogenesis, the expression of miR204 was then analyzed by using qPCR. Compared with the normal control BMSCs, BMSCs $^{- \text {T2DM }}$ had approximately ninefold increase of miR204 (Figure 1C). Moreover, miR204 decreased upon 10-day osteogenic induction, while the expression in $\mathrm{BMSCs}^{- \text {T2DM }}$ remained high (Figure 1D). All of these data implicate that miR204 might contribute to the osteogenic defect in the 
A

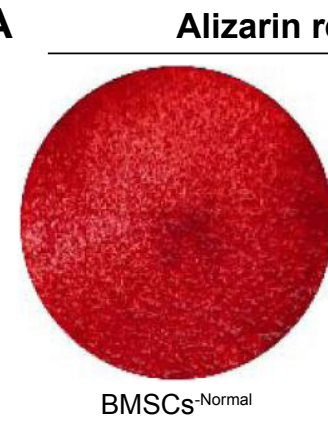

BMSCs $^{- \text {Normal }}$

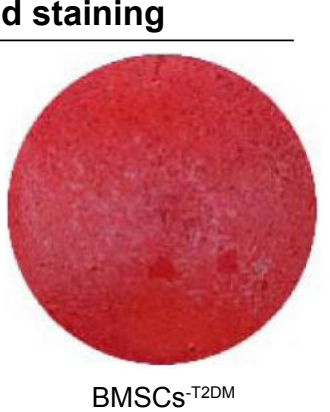

B
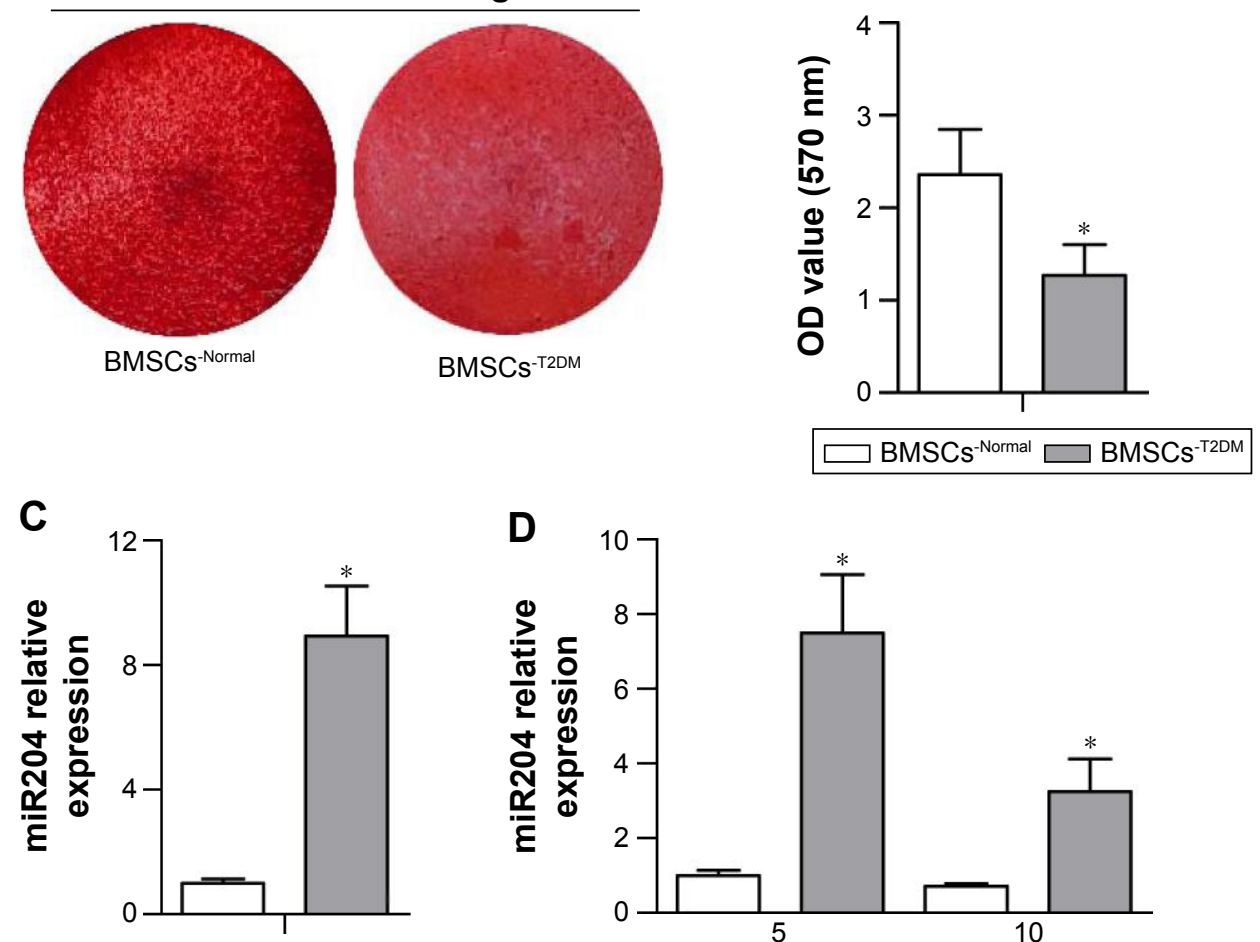

D

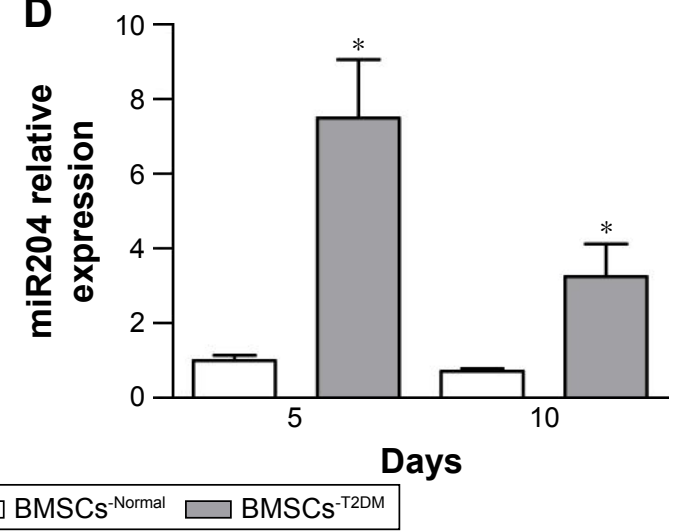

Figure I Upregulation of miR204 contributes to the decreased osteogenesis in the BMSCs of T2DM. Alizarin red staining of extracellular matrix mineralization of osteogenic differentiated BMSCs and BMSCs ${ }^{-T_{2 D M}}$ (A) and quantitative data (B). miR204 expression in undifferentiated BMSCs and BMSCs ${ }^{-{ }^{T 2 D M}}$ (C). miR204 expression in BMSCs and $\mathrm{BMSCs}^{-\mathrm{T} 2 \mathrm{DM}}$ upon osteogenic induction (D).

Note: $* p<0.05$ versus normal.

Abbreviations: T2DM, type 2 diabetes mellitus; BMSCs, bone marrow stem cells; OD, optical density.

$\mathrm{BMSCs}^{-\mathrm{T} 2 \mathrm{DM}}$ and that blocking miR204 would promote the osteogenetic ability of $\mathrm{BMSCs}^{-\mathrm{T} 2 \mathrm{DM}}$.

\section{Surface characterization of titanium implant delivery system}

To deliver miRNA inhibitors from the titanium implant surface, the implant was then functionalized as described in the "Materials and methods" section. As shown in the SEM images (Figure 2A), the surface layer of original MAO titanium implant was covered by a large number of micropores ranging from 1 to $10 \mu \mathrm{m}$. For miRNA inhibitor delivery, the implant was covered with an ultrathin layer of PLGA membrane (PLGA sheet). Notably, the PLGA membrane integrated tightly with the implant by penetrating into the micropores (Figure 2B and C). Moreover, the uniform appearance of the PLGA sheet surface could be observed, with plenty of microgrooves and pits among them due to the evaporation of the solvent DCM (Figure 2D). These existing microgrooves and pits may function as the release channel for miRNA delivery when the implant is placed in the body.

\section{Distribution of AuNP-antagomiRNAs in the PLGA sheet}

Ultrathin sections of the PLGA sheets were processed for TEM analysis, and the gold nanoparticles $\sim 15-20 \mathrm{~nm}$ (miRNA-conjugated AuNP would be larger than the original size) in diameter were seen dispersed evenly throughout the sheet (Figure 3). Accordingly, amounts of fluorescent spots indicating labeled nucleic acids were found to be spread around the PLGA sheets by three-dimensional LSCM analysis (Figure 4A and E), which revealed even distribution of gold nanoparticles in the PLGA sheets.

\section{Release and uptake of AuNP- antagomiRNAs from PLGA sheets}

BMSCs were seeded on the titanium disc functionalized with PLGA-AuNP-Cy3-labeled antagomiR204 sheet. After 

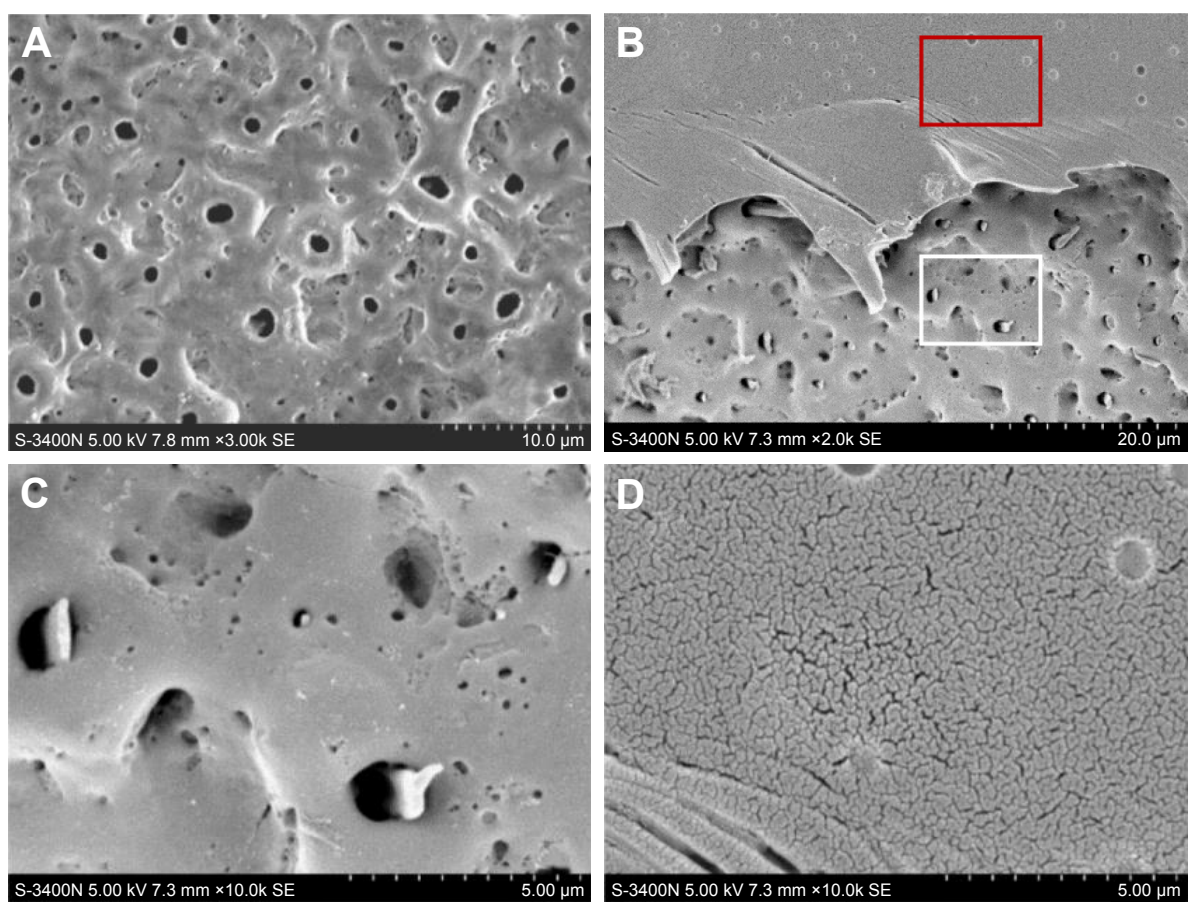

Figure 2 Surface morphology of original MAO titanium implant and MAO titanium implant with PLGA coating. Representative SEM image of the original MAO titanium implant surface (A). The border between PLGA coating and titanium implant (B). High magnification image of the white rectangle area in B showing the PLGA penetrated into the micropores of MAO implant (C). High magnification image of the red rectangle area in B displaying the surface of PLGA coating (D).

Abbreviations: SEM, scanning electron microscopy; MAO, microarc oxidation; PLGA, poly(lactic-co-glycolic acid).

24 hours, the cells were fixed and Cy3 fluorescence was observed under LSCM. Accumulating fluorescent signals were found to be spread around the nuclei (Figure 5), indicating that the gold nanoparticles conjugated with miR204-inhibitor were successfully released from the PLGA sheet and uptake by BMSCs ${ }^{-T 2 D M}$.

\section{Cytotoxicity assay and cell adhesion}

The cytotoxicity of constructed delivery system was determined by analyzing the survival and proliferation of cells

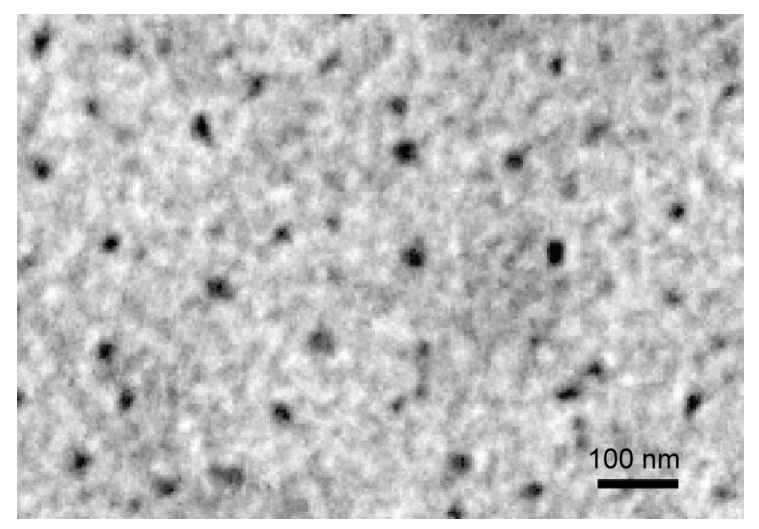

Figure 3 Representative TEM image showing the distribution of gold nanoparticles in PLGA coating.

Abbreviations: TEM, transmission electron microscopy; PLGA, poly(lactic-coglycolic acid). seeded. The cell population displayed a similar growth curve over a 4-day monitoring period among titanium only, PLGA with control and PLGA with miR204 inhibitor (Figure 6A), suggesting that the PLGA delivery system-functionalized titanium had no obvious effects on the cell survival and proliferation. In addition, the PLGA delivery systemfunctionalized titanium did not change the adhesion performance of seeded BMSCs, as indicated by the similar adhesive cell numbers 4 hours after seeding (Figure 6B and C).

\section{In vitro effects of PLGA delivery system on osteogenesis}

Next, the effects of PLGA sheet-mediated delivery of miR204 inhibitor on osteogenesis by analyzing key marker Alp activity in the early stage of osteogenetic differentiation were studied. The BMSCs $^{\text {T2DM }}$ seeded on PLGA with miR204-inhibitor-conjugated AuNP had significant improvement of Alp activity upon osteogenic induction (Figure 7A). Accordingly, the alizarin red staining also confirmed the osteogenesis-promoting effects of PLGA with miR204 inhibitor-conjugated AuNP (Figure 7B and C).

Besides ALP activity and alizarin red staining, the expression of osteogenesis-specific genes including Bmp, Opg, Alp, Runx2, and Col I during 10-day induced differentiation of $\mathrm{BMSCs}^{-\mathrm{T} 2 \mathrm{DM}}$ seeded on the PLGA drug delivery 

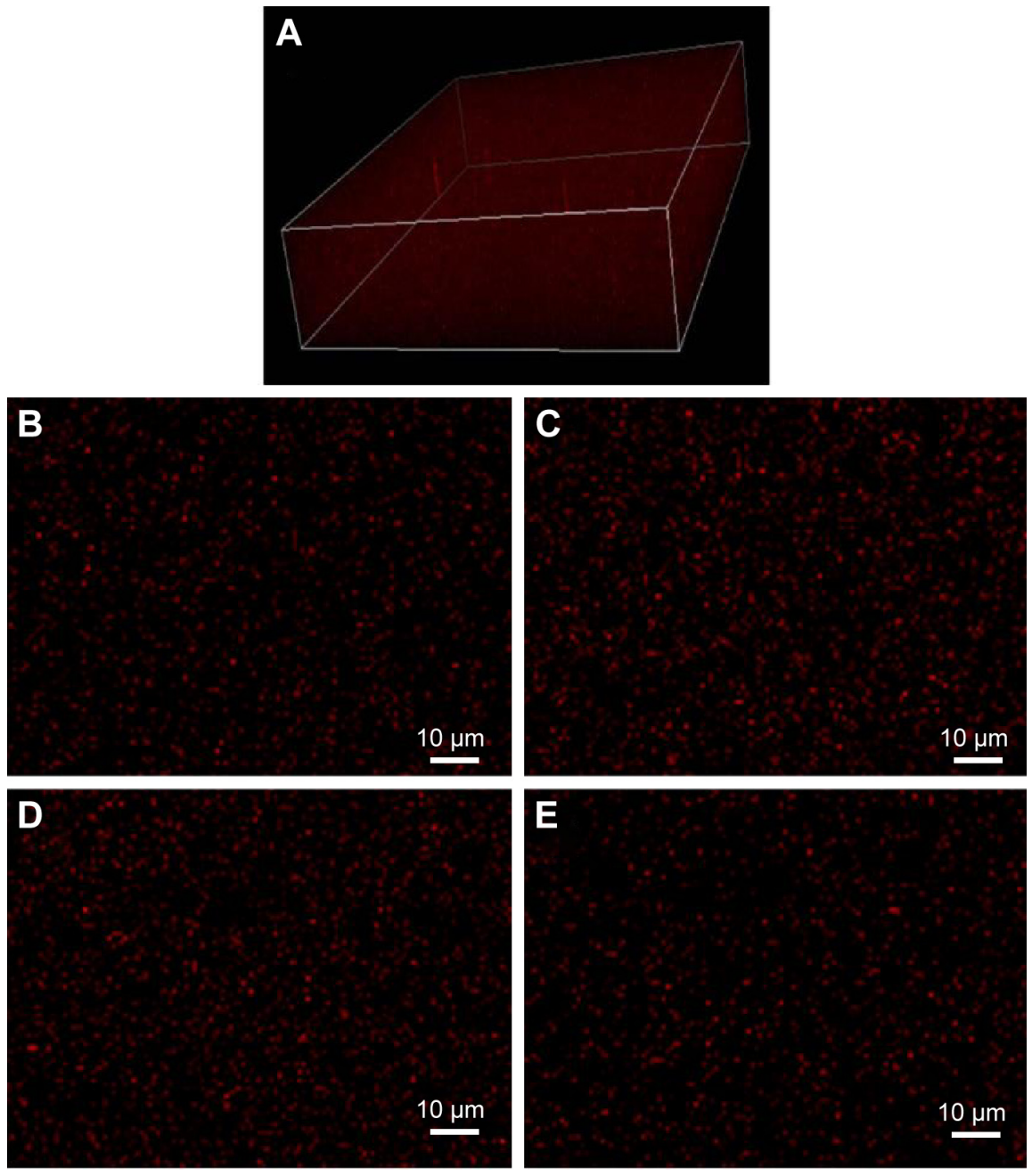

Figure 4 3D distribution of Cy3-labeled miRNA inhibitor in PLGA coating. Cy3-labeled miRNA inhibitor was conjugated to AuNP and the AuNP was dispersed into the PLGA solution before sheet making. The resultant PLGA-AuNP-miRNA inhibitor sheet was scanned by confocal laser scan microscope. 3D reconstruction of fluorescencelabeled miRNA inhibitor in PLGA sheet (A). Plane images to show the distribution of the labeled AuNP from surface to inside of the PLGA coating; the plane image was harvested approximately every eight $\mu \mathrm{m}$ (B-E).

Abbreviations: 3D, three-dimensional; AuNP, gold nanoparticle; miRNA, microRNA; PLGA, poly(lactic-co-glycolic acid).

system armed with either control or miR204-inhibitor was also investigated. In general, the miR204 inhibitor delivery significantly increased the osteogenic gene expression, compared with the scramble inhibitor delivery (Figure 7D and $\mathrm{H})$. Specifically, the abundance of gene expression was increased by two- to fourfold at day 5 of induction, and the fold was further augmented to 6-10 times at day 10 upon osteogenic induction (Figure 7D and $\mathrm{H}$ ). These preliminary in vitro results further suggest the applicability of this system in improving the osteogenic capability of BMSC-T2DM.
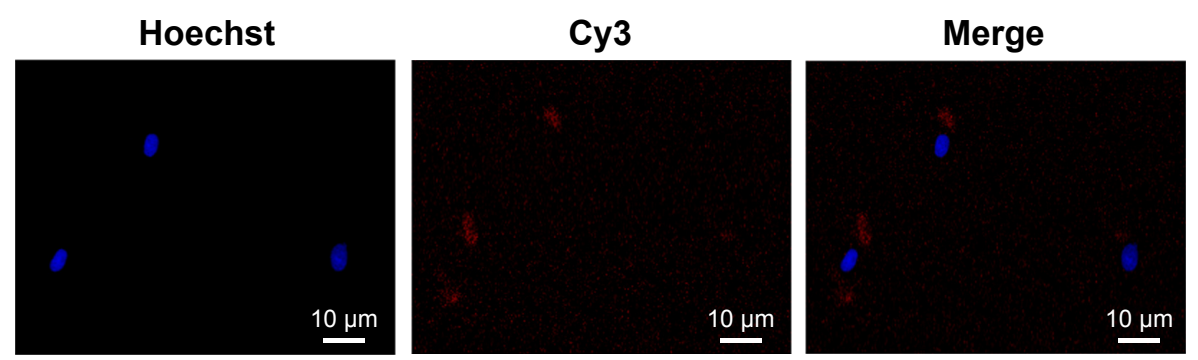

Figure 5 Uptake of the miRNA-AuNP in the PLGA sheet by seeded cells. BMSCs were seeded on the PLGA sheet. Uptake of the miRNA-AuNP was monitored by confocal laser scan microscope.

Abbreviations: AuNP, gold nanoparticles; BMSCs, bone marrow stem cells; miRNA, microRNA; PLGA, poly(lactic-co-glycolic acid). 
A

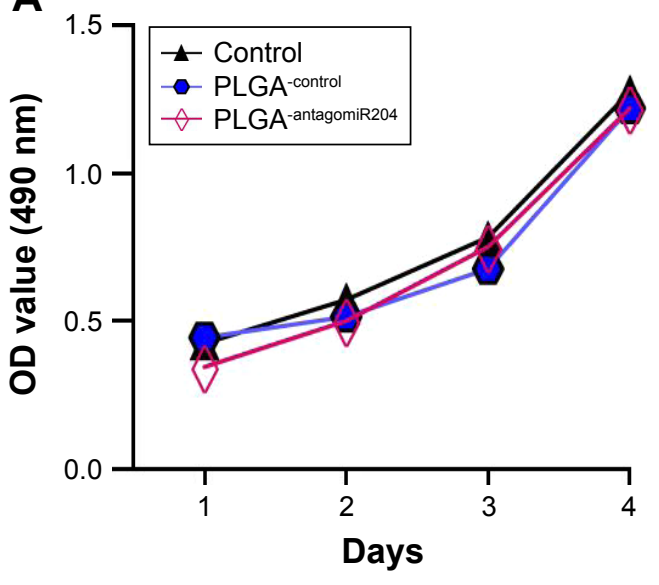

B

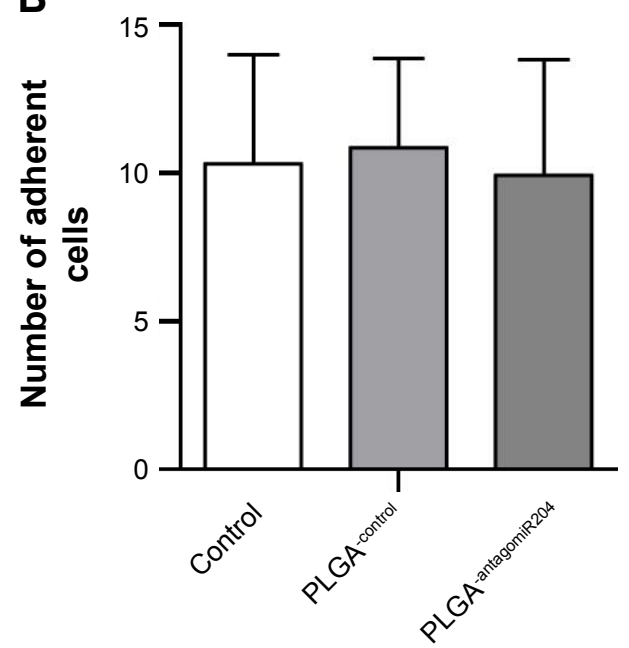

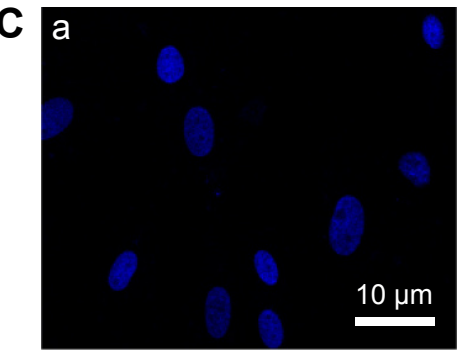
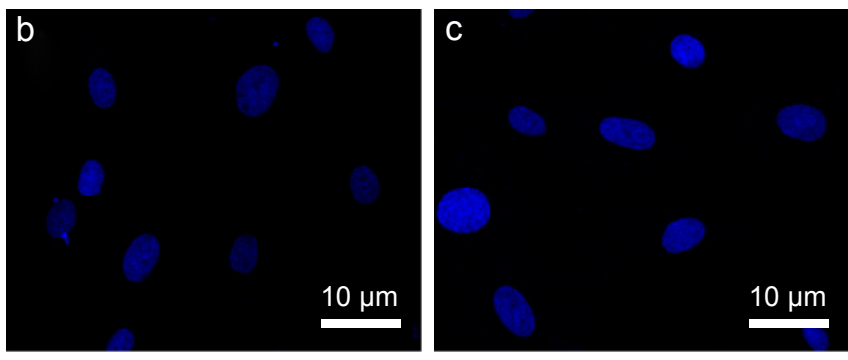

Figure 6 Cell viability determined by CCK8 $(\mathbf{A})$ and adherent cell numbers determined by counting the Hoechst-stained nuclei (B and $\mathbf{C})$. Notes: (C-a): Control; (C-b): PLGA ${ }^{\text {-control; }}$ (C-c): PLGA-antagomir204.

Abbreviations: CCK8, cell counting kit 8; OD, optical density; PLGA, poly(lactic-co-glycolic acid).

\section{Critical removal torque analysis}

None of the PLGA films on the titanium implants were found dropped off in the course of implanting, confirming the tight integration of the PLGA film with the titanium and making it possible to release AuNP in the following weeks. After an 8 -week healing phase, the implant samples combined with the intact tibia were harvested, and the osseointegration condition was examined by critical removal torque assay. The critical removal torque for implants functionalized with PLGA sheet only was similar to original MAO ones (Figure 8). Moreover, the implants functionalized with PLGA sheet together with miR204-inhibitor showed a higher critical removal torque value than the PLGA only or PLGA with scramble miRNA inhibitor in T2DM rats (Figure 8).

\section{MicroCT reconstruction of osseointegration}

In addition, the osseointegration of implants was evaluated by microCT scanning followed by three-dimensional reconstruction. The peri-implant trabecular microstructures and the correlated parameters are shown in Figure 9A and D. As expected, there were relatively few trabecular microstructures around the titanium implant without functionalization or with PLGA sheet armed with scramble miRNA inhibitor (Figure 9A). By contrast, implants functionalized with PLGA sheet armed with miR204 inhibitor were almost fully covered by trabeculae of different sizes (Figure 9A). The qualitative analysis of parameters, including bone tissue fraction, trabecular number, and trabecular spacing, further demonstrated the beneficial effects of the PLGA sheet-based delivery of miR204 inhibitor (Figure 9B and D).

\section{Histological evaluation}

After hard tissue section and processing, Van-Gieson staining was done to provide more accurate and detailed features regarding osseo-implant interface. Newly formed bone and titanium screw were clearly exhibited, and the implants functionalized with PLGA sheet armed with miR204 inhibitor had significantly more bone structures in comparison with the controls (Figure 10A). Approximately $20 \%$ more BIC rate was found in implants receiving miR204 inhibitor delivery than the other two groups (Figure 10B).

\section{Discussion}

In this study, the authors have identified that miR204 was one of the key mediators responsible for the impaired osteogenesis 


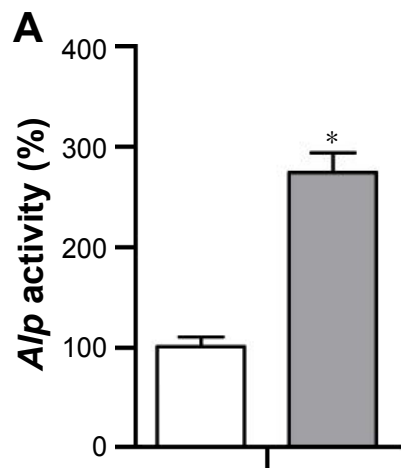

C

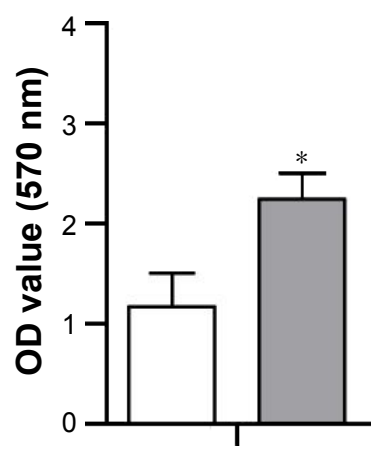

E

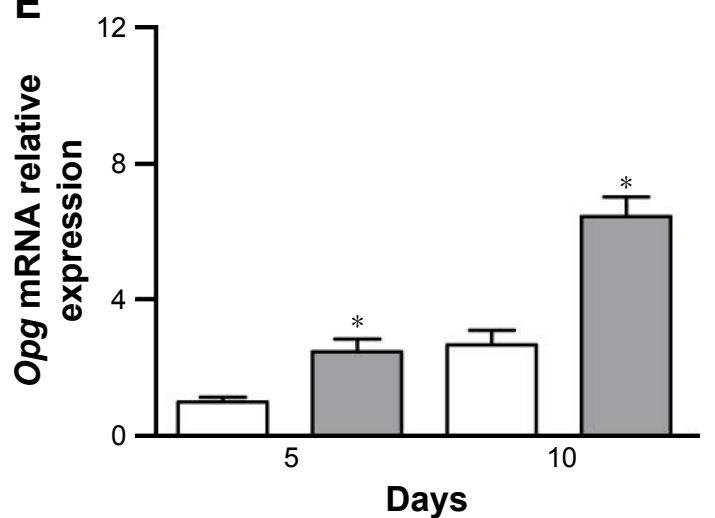

G

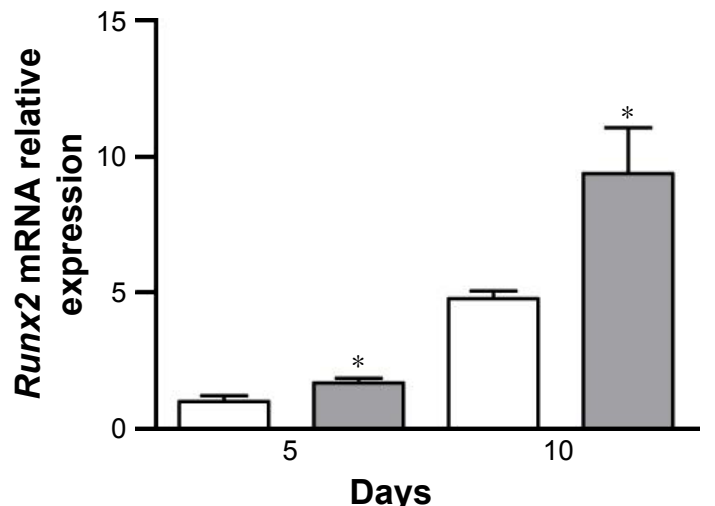

B

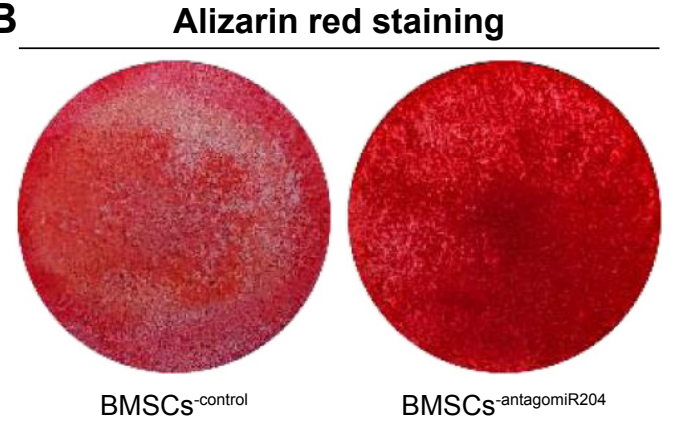

D

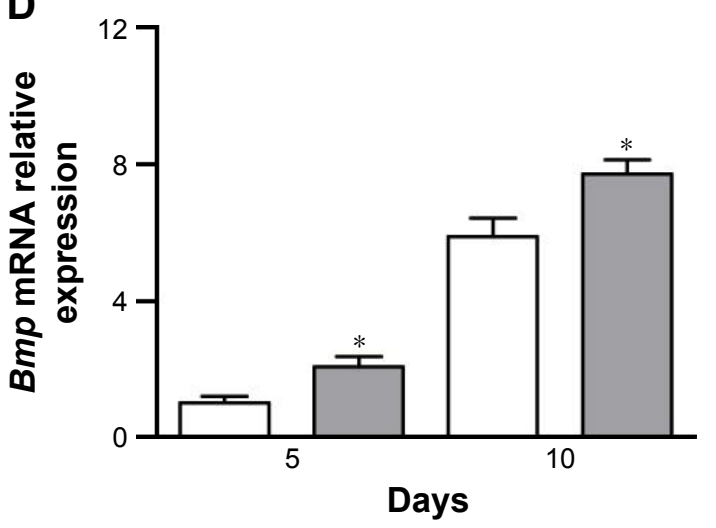

$\mathbf{F}$

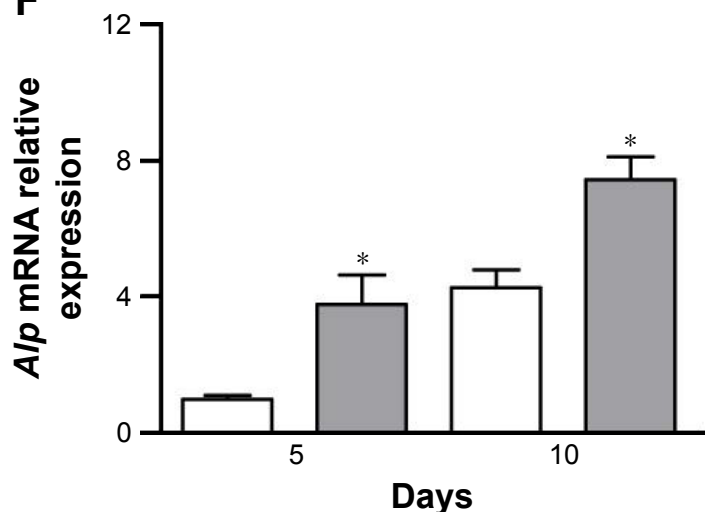

H

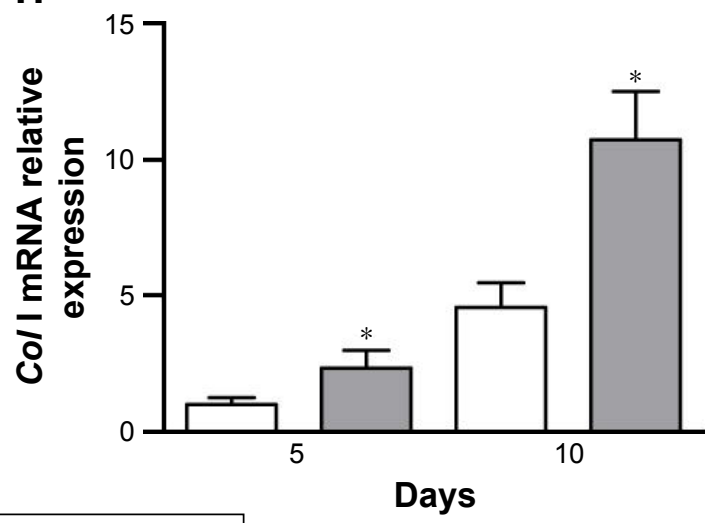

Figure 7 Alkaline phosphatase activity assay $(\mathbf{A})$ and alizarin red staining $(\mathbf{B})$, quantitative analysis of the alizarin red staining $(\mathbf{C})$ of BMSCs $^{\text {-control }}$ and BMSC $^{\text {-antagomiR204. }}$ Osteogenic-related gene expression determined by RT-PCR after 5 or 10 days osteogenic induction, including Bmp (D), Opg (E), Alp (F), Runx2 (G), and Col I (H). Note: $* P<0.05$ versus BMSCs sontrol. $^{\text {co }}$

Abbreviations: BMSCs, bone marrow stem cells; mRNA, messenger RNA; OD, optical density; RT-PCR, reverse transcription-polymerase chain reaction; Bmp, bone morphogenetic protein; Opg, osteoprotegerin; AIP, alkaline phosphatase; Runx2, runt-related transcription factor 2; Col I, collagen type I. 


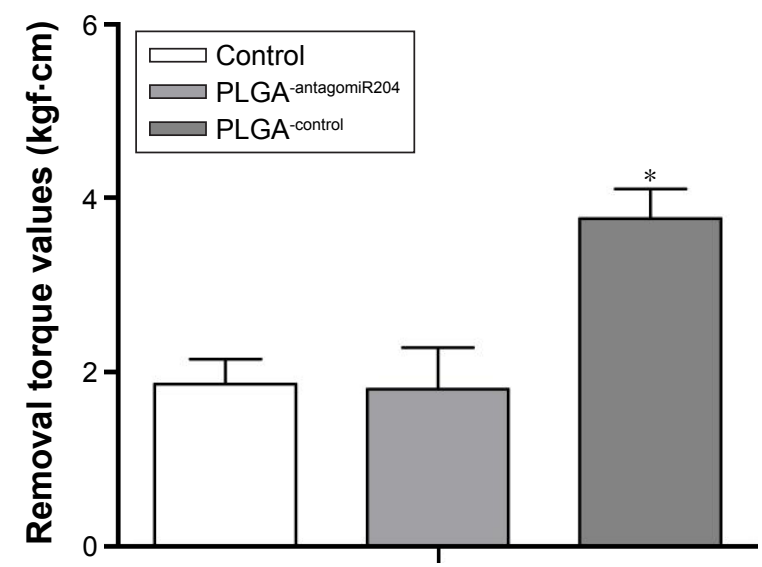

Figure 8 Removal torque value for the implant samples. Note: ${ }^{* P}<0.05$ versus control and PLGA-control.

Abbreviation: PLGA, poly(lactic-co-glycolic acid).

observed in T2DM. Moreover, a PLGA-based miR204 delivery system for the titanium implant was designed, which promotes osseointegration in diabetic rat model via promoting BMSCs osteogenesis in vivo. The established strategy might be promising in the future clinical application.

\section{miR204 acts as an inhibitor of osteogenesis in DM}

Patients with DM have bad healing outcomes upon bone injury and a relatively low success rate of dental implantation in stomatology scopes. ${ }^{4,24,25}$ Although intensive studies have focused on how DM results in worse bone injury recovery and poorer bone-implant osseointegration, limited achievements had been gained, not even to say the targeted therapeutic strategies. It is generally accepted that the phenotype observed in DM patients is at least partially attributed to the deficient osteogenesis. ${ }^{7,17}$ Identification of the key molecules responsible for the defect would open an avenue for therapy. As therapeutic targets, miRNAs are of priority due to not only its potent function but also the feasibility for drug delivery and targeting. ${ }^{26}$ Previously, multiple miRNAs are found to be closely related to bone development and metabolism ${ }^{27}$ and play an important role in promoting new bone regeneration, ${ }^{28}$ such as miR218, miR27, miR204, and miR223. ${ }^{20,29-31}$ These miRNAs serve as regulators of mineral deposition through acting as drivers or inhibitors of bone-related signaling pathways. Specifically, miR204 has been revealed to negatively regulate the essential osteogenic transcription factor Runx $2 .{ }^{20}$ This study further confirmed the negative regulatory role of miR204 on osteogenesis. Through comparing the expression characteristics of miR204 during $\mathrm{BMSCs}^{\text {-T2DM }}$ osteogenic differentiation, increased miR204 was identified as a determinant for deficient osteogenic capacity of BMSCs derived from T2DM rats. Notably, the detailed mechanism of how misexpressed miR204 inhibited osteogenesis is not fully

\section{A}
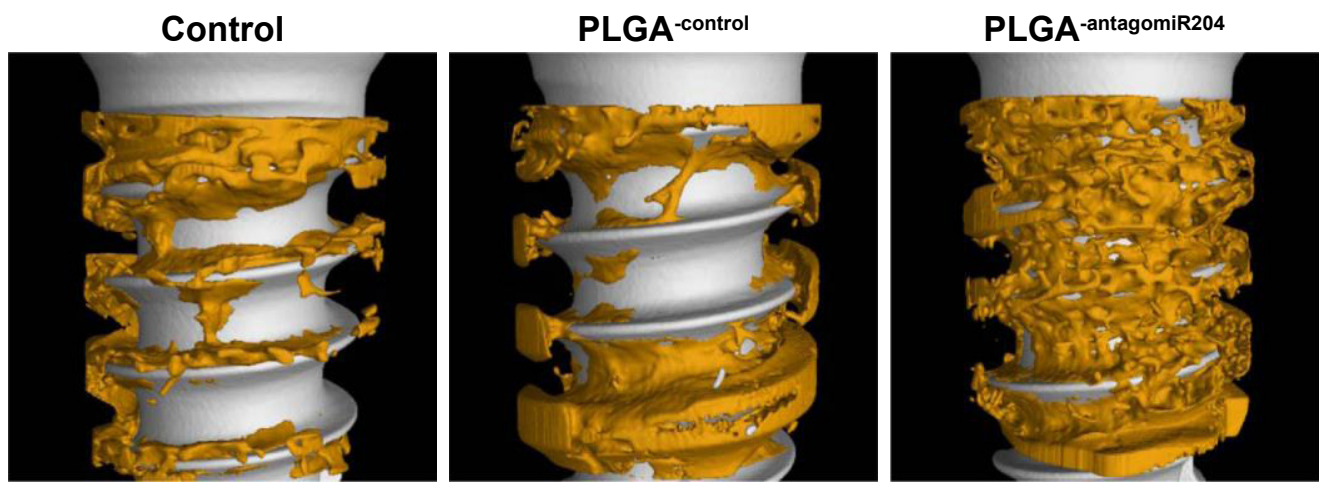

B

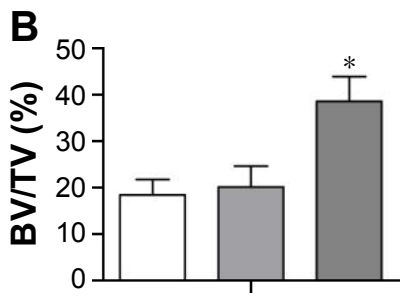

C

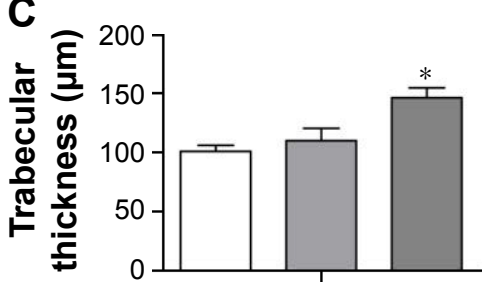

D

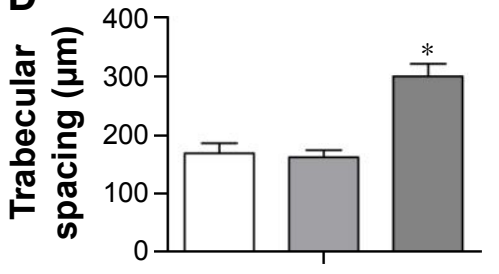

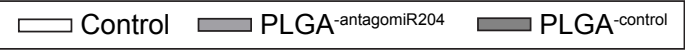

Figure 9 3D reconstruction of the microCT scan results displaying the bone (yellow) surrounding implant (gray) at 8 weeks after implantation (A). Quantitative analysis of BV/TV (B), trabecular thickness (C), and trabecular spacing (D).

Note: ${ }^{*} \boldsymbol{P}<0.05$ versus control and PLGA-control.

Abbreviations: 3D, three-dimensional; microCT, microcomputerized tomography; PLGA, poly(lactic-co-glycolic acid); BV, bone volume; TV, total volume. 
A

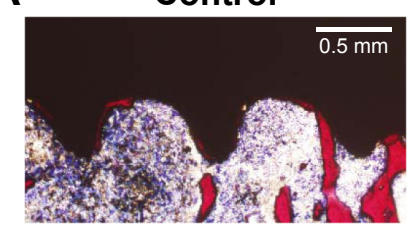

PLGA-control

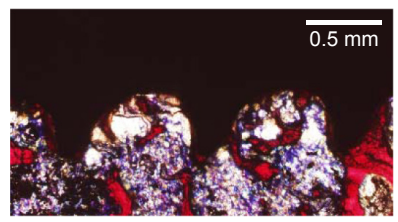

PLGA-antagomiR204

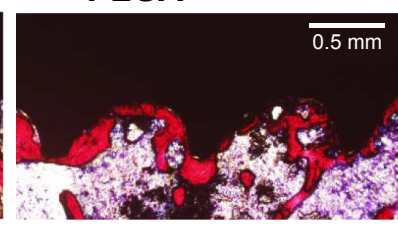

B

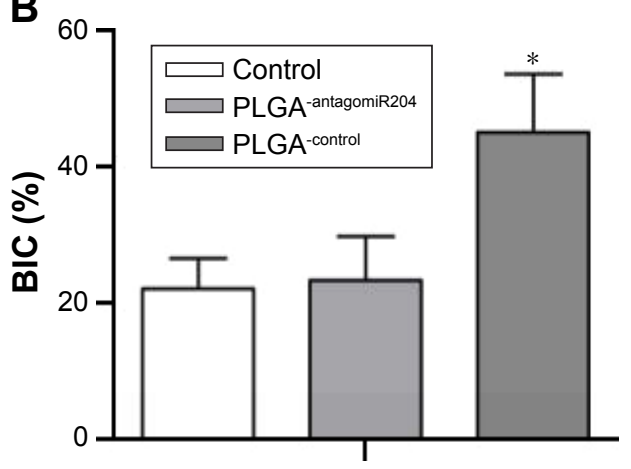

Figure 10 Van-Gieson staining images displaying the bone (red) attached to implant (black) at 8 weeks after implantation of different surface functionalized implants (A) and quantitative analysis of BIC (B).

Note: $* P<0.05$ versus control and PLGA-control.

Abbreviations: PLGA, poly(lactic-co-glycolic acid); BIC, bone-implant contact.

understood. Future work is needed to clarify other targets besides Runx2 involved in osteogenesis inhibition.

\section{PLGA-based miRNA delivery system in implant functionalization}

In terms of miRNA/siRNA delivery, various RNA carriers have been developed and applied under experimental conditions, including liposomes, exosomes, and gold nanoparticles. ${ }^{32-34}$ However, there were few studies applying these nanoparticles to titanium implant, mainly due to lack of the strategies to load and preserve the nanoparticles on the surface of the titanium implant. For example, implants have been modified with miR29b and antimiR 138 by lyophilizing miRNA lipoplexes onto microporous titanium surface. ${ }^{35}$ However, the high affinity of lipoplexes to the titanium surface and the stability of lyophilized miRNAs are both the hurdle for efficient drug delivery. Recently, miRNA-21 chitosan/hyaluronic acid nanoparticles were developed, which can be stably loaded to MAO titanium implant surface with newly developed gel coating ${ }^{36}$ and thus overcomes some of the problems. In this study, miRNAconjugated gold nanoparticles into the PLGA sheet were dispersed, which released the intact gold nanoparticles for the adherent cells. The rational release of siRNA-conjugated AuNP mainly relies on the following: 1) the thin PLGA sheet could stably attach the modified titanium implant, which remains intact after in vivo transplantation; and 2) the gradual degradation of PLGA releases embedded AuNP.
The combinatory relatively high stability on the implant and gradual degradation rate make PLGA a rational carrier for releasing AuNP. Moreover, PLGA is approved by the United States Food and Drug Administration (FDA) for clinical use in human beings, ${ }^{37}$ and a lot of drug regents based on PLGA delivery system come forth for the treatment of various diseases ${ }^{38}$ This study further clarified that PLGA is compatible with AuNP-mediated siRNA delivery, particularly in the field of dental implantation. Furthermore, the strategy proposed is of favorable biocompatibility, easy to manipulate, and of controllable biodegradation rate. Taken together, it was demonstrated that PLGA sheet dispersed with miR204-inhibitor-conjugated AuNP could actually deliver the miRNA inhibitor for improvement of osseointegration in the context of T2DM for dental implantation. These preliminary findings suggest a potential and potent therapeutic miRNA/siRNA delivery strategy to better the prognosis of T2DM implantation.

\section{Conclusion}

This study for the first time has proposed that the misexpression of miR204 accounted for the impaired osteogenesis in T2DM and miR204 inhibition indeed improved the osteogenic differentiation capacity of BMSCs derived from T2DM rats. Moreover, a PLGA-based system for the delivery of antagomiR204-conjugated AuNP from the implant was established, which substantially promoted osseoimplantation in T2DM rats. Altogether, this study provides a promising 
strategy for implant surface functionalization to overcome poor bone-implant contact in T2DM.

\section{Acknowledgment}

The study was supported by the Natural Science Foundation of China (NSFC grants: 81470775, 81170984, 81271579, and 81300918).

\section{Disclosure}

The authors report no conflicts of interest in this work.

\section{References}

1. van Velzen FJJ, Ofec R, Schulten EAJM, ten Bruggenkate CM. 10-year survival rate and the incidence of peri-implant disease of 374 titanium dental implants with a SLA surface: a prospective cohort study in 177 fully and partially edentulous patients. Clin Oral Implants Res. 2015;26(10):1121-1128.

2. Charyeva O, Altynbekov K, Zhartybaev R, Sabdanaliev A. Long-term dental implant success and survival - a clinical study after an observation period up to 6 years. Swed Dent J. 2012;36(1):1-6.

3. Marchand F, Raskin A, Dionnes-Hornes A, et al. Dental implants and diabetes: conditions for success. Diabetes Metab. 2012;38(1):14-19.

4. Annibali S, Pranno N, Cristalli MP, La Monaca G, Polimeni A. Survival analysis of implant in patients with diabetes mellitus: a systematic review. Implant Dent. 2016;25(5):663-674.

5. International Diabetes Federation. IIDF Diabetes Atlas - 7th edition. Available from: http://www.diabetesatlas.org/resources/2015-atlas. html. Accessed October 1, 2016.

6. Yang W, Lu J, Jia W, Zhang L. Prevalence of diabetes among men and women in China. N Engl J Med. 2010;362(12):1090-1101.

7. Napoli N, Chandran M, Pierroz DD, et al. Mechanisms of diabetes mellitus-induced bone fragility. Nat Rev Endocrinol. 2017;13(4): 208-219.

8. Merlotti D, Gennari L, Dotta F, Lauro D, Nuti R. Mechanisms of impaired bone strength in type 1 and 2 diabetes. Nutr Metab Cardiovasc Dis. 2010;20(9):683-690.

9. Al Amri MD, Abduljabbar TS. Comparison of clinical and radiographic status of platform-switched implants placed in patients with and without type 2 diabetes mellitus: a 24-month follow-up longitudinal study. Clin Oral Implants Res. 2017;28(2):226-230.

10. de Souza Bastos A, Graves DT, de Melo Loureiro AP, et al. Diabetes and increased lipid peroxidation are associated with systemic inflammation even in well-controlled patients. J Diabetes Complications. 2016; 30(8):1593-1599.

11. Smeets R, Stadlinger B, Schwarz F, et al. Impact of dental implant surface modifications on osseointegration. BioMed Res Int. 2016; 2016:6285620.

12. Sammartino G, Dohan Ehrenfest DM, Shibli JA, Galindo-Moreno P. Tissue engineering and dental implantology: biomaterials, new technologies, and stem cells. BioMed Res Int. 2016;2016:5713168.

13. Wang L, Zou D, Zhang S, Zhao J, Pan K, Huang Y. Repair of bone defects around dental implants with bone morphogenetic protein/fibroblast growth factor-loaded porous calcium phosphate cement: a pilot study in a canine model. Clin Oral Implants Res. 2011;22(2):173-181.

14. Shen X, Zhang Y, Gu Y, et al. Sequential and sustained release of SDF-1 and BMP-2 from silk fibroin-nanohydroxyapatite scaffold for the enhancement of bone regeneration. Biomaterials. 2016;106: 205-216.

15. Pritchard CC, Cheng HH, Tewari M. MicroRNA profiling: approaches and considerations. Nat Rev Genet. 2012;13(5):358-369.

16. Jonas $S$, Izaurralde $E$. Towards a molecular understanding of microRNAmediated gene silencing. Nat Rev Genet. 2015;16(7):421-433.
17. Epstein S, Defeudis G, Manfrini S, et al. Diabetes and disordered bone metabolism (diabetic osteodystrophy): time for recognition. Osteoporosis Int. 2016;27(6):1931-1951.

18. MacNabb C, Patton D, Hayes JS. Sclerostin antibody therapy for the treatment of osteoporosis: clinical prospects and challenges. J Osteoporos. 2016;2016:6217286.

19. Mafi Golchin M, Heidari L, Ghaderian SM, Akhavan-Niaki H. Osteoporosis: a silent disease with complex genetic contribution. $J$ Genet Genomics. 2016;43(2):49-61.

20. Huang J, Zhao L, Xing L, Chen D. MicroRNA-204 regulates Runx2 protein expression and mesenchymal progenitor cell differentiation. Stem Cells. 2010;28(2):357-364.

21. Zheng D, Giljohann DA, Chen DL, et al. Topical delivery of siRNAbased spherical nucleic acid nanoparticle conjugates for gene regulation. Proc Natl Acad Sci U S A. 2012;109(30):11975-11980.

22. Ribot J, Caliaperoumal G, Paquet J, Boisson-Vidal C, Petite H, Anagnostou F. Type 2 diabetes alters mesenchymal stem cell secretome composition and angiogenic properties. J Cell Mol Med. 2017;21(2): 349-363.

23. Shin L, Peterson DA. Impaired therapeutic capacity of autologous stem cells in a model of type 2 diabetes. Stem Cells Transl Med. 2012; 1(2):125-135.

24. Devlin H, Garland H, Sloan P. Healing of tooth extraction sockets in experimental diabetes mellitus. J Oral Maxillofac Surg. 1996;54(9): 1087-1091.

25. Taylor GW, Burt BA, Becker MP, et al. Non-insulin dependent diabetes mellitus and alveolar bone loss progression over 2 years. J Periodontol. 1998;69(1):76-83.

26. Peng B, Chen Y, Leong KW. MicroRNA delivery for regenerative medicine. Advanced Drug Deliv Rev. 2015;88:108-122.

27. Dole NS, Delany AM. MicroRNA variants as genetic determinants of bone mass. Bone. 2016;84:57-68.

28. Lian JB, Stein GS, van Wijnen AJ, et al. MicroRNA control of bone formation and homeostasis. Nat Rev Endocrinol. 2012;8(4):212-227.

29. Park MG, Kim JS, Park SY, et al. MicroRNA-27 promotes the differentiation of odontoblastic cell by targeting APC and activating Wnt/ beta-catenin signaling. Gene. 2014;538(2):266-272.

30. van Wijnen AJ, van de Peppel J, van Leeuwen JP, et al. MicroRNA functions in osteogenesis and dysfunctions in osteoporosis. Curr Osteoporos Rep. 2013;11(2):72-82.

31. Sun MG, Zhou XY, Chen LL, et al. The regulatory roles of microRNAs in bone remodeling and perspectives as biomarkers in osteoporosis. BioMed Res Int. 2016;2016:1652417.

32. Ghosh R, Singh LC, Shohet JM, Gunaratne PH. A gold nanoparticle platform for the delivery of functional microRNAs into cancer cells. Biomaterials. 2013;34(3):807-816.

33. Xu HP, Li ZY, Si J. Nanocarriers in gene therapy: a review. J Biomed Nanotechnol. 2014;10(12):3483-3507.

34. Tominaga N, Yoshioka Y, Ochiya T. A novel platform for cancer therapy using extracellular vesicles. Adv Drug Deliv Rev. 2015;95:50-55.

35. Wu K, Song W, Zhao L, et al. MicroRNA functionalized microporous titanium oxide surface by lyophilization with enhanced osteogenic activity. ACS Appl Mater Interfaces. 2013;5(7):2733-2744.

36. Wang Z, Wu G, Feng Z, et al. Microarc-oxidized titanium surfaces functionalized with microRNA-21-loaded chitosan/hyaluronic acid nanoparticles promote the osteogenic differentiation of human bone marrow mesenchymal stem cells. Int J Nanomed. 2015;10:6675-6687.

37. Wang H, Agarwal P, Zhao S, et al. Hyaluronic acid-decorated dual responsive nanoparticles of Pluronic F127, PLGA, and chitosan for targeted co-delivery of doxorubicin and irinotecan to eliminate cancer stem-like cells. Biomaterials. 2015;72:74-89.

38. Xu Y, Kim CS, Saylor DM, Koo D. Polymer degradation and drug delivery in PLGA-based drug-polymer applications: a review of experiments and theories. J Biomed Materials Res Part B Appl Biomater. Epub April 21, 2016. 
International Journal of Nanomedicine

Dovepress

\section{Publish your work in this journal}

The International Journal of Nanomedicine is an international, peerreviewed journal focusing on the application of nanotechnology in diagnostics, therapeutics, and drug delivery systems throughou the biomedical field. This journal is indexed on PubMed Central, MedLine, CAS, SciSearch ${ }^{\circledR}$, Current Contents ${ }^{\circledR} /$ Clinical Medicine,
Journal Citation Reports/Science Edition, EMBase, Scopus and the Elsevier Bibliographic databases. The manuscript management system is completely online and includes a very quick and fair peer-review system, which is all easy to use. Visit http://www.dovepress.com/ testimonials.php to read real quotes from published authors.

Submit your manuscript here: http://www.dovepress.com/international-journal-of-nanomedicine-journal 